\title{
PERFORMANCE VALIDATION OF HIGH RESOLUTION DIGITAL SURFACE MODELS GENERATED BY DENSE IMAGE MATCHING WITH THE AERIAL IMAGES
}

\author{
N. Yastikli ${ }^{\mathrm{a},}{ }^{*}$, H. Bayraktar ${ }^{\mathrm{b}}$ and Z. Erisir ${ }^{\mathrm{a}}$ \\ ${ }^{a}$ YTU, Civil Engineering Faculty, Department of Geomatics Engineering, 34210 Davutpasa, Istanbul, Turkey- \\ (ynaci,zerisir)@yildiz.edu.tr \\ ${ }^{\mathrm{b}}$ General Directorate of Geographical Information Systems, Ministry of Environment and Urbanism, 06510, Balgat, Ankara, Turkey- \\ huseyin.bayraktar@csb.gov.tr
}

Commission I, WG I/4

KEY WORDS: Performance, Analyses, DSM, Image, Matching, Photogrammetry

\begin{abstract}
:
The digital surface models (DSM) are the most popular products to determine visible surface of Earth which includes all non-terrain objects such as vegetation, forest, and man-made constructions. The airborne light detection and ranging (LiDAR) is the preferred technique for high resolution DSM generation in local coverage. The automatic generation of the high resolution DSM is also possible with stereo image matching using the aerial images. The image matching algorithms usually rely on the feature based matching for DSM generation. First, feature points are extracted and then corresponding features are searched in the overlapping images. These image matching algorithms face with the problems in the areas which have repetitive pattern such as urban structure and forest.

The recent innovation in camera technology and image matching algorithm enabled the automatic dense DSM generation for large scale city and environment modelling. The new pixel-wise matching approaches are generates very high resolution DSMs which corresponds to the ground sample distance (GSD) of the original images. The numbers of the research institutes and photogrammetric software vendors are currently developed software tools for dense DSM generation using the aerial images. This new approach can be used high resolution DSM generation for the larger cities, rural areas and forest even Nation-wide applications. In this study, the performance validation of high resolution DSM generated by pixel-wise dense image matching in part of Istanbul was aimed. The study area in Istanbul is including different land classes such as open areas, forest and built-up areas to test performance of dense image matching in different land classes. The obtained result from this performance validation in Istanbul test area showed that, high resolution DSM which corresponds to the ground sample distance (GSD) of original aerial image can be generated successfully by pixel-wise dense image matching using commercial and research institution's software.
\end{abstract}

\section{INTRODUCTION}

The digital representation of the Earth surface in three dimensions (3D) has vital importance in wide range of applications such as urban planning and management, disaster monitoring, agriculture, forestry and hydrology. The digital topographic data of the Earth surface is represented by digital terrain models (DTMs), digital elevation models (DEMs) and digital surface models (DSMs). The DTM and DEM are describing the bare Earth surface but only difference between them is additional information on DTM such as break-lines and mass points. DSM is describing the visible surface of Earth including all non-terrain objects such as vegetation, forest, and man-made constructions.

The DSM and DEM are the most common and demanded products and different techniques are employed for production of them. The space-borne optical stereoscopy (Li et al., 2002; Kaczynski et al., 2004; Toutin, 2002; Toutin, 2008) and spaceborne interferometric synthetic aperture radar (InSAR) (Sefercik and Yastikli, 2014; Sefercik et al., 2014) are the techniques for the DSM an DEM generation with the low cost, time saving processing and global coverage. The first product of spaceborne remote sensing techniques is a DSM. The DEM is obtained by removing non-terrain objects (Jacobsen, 2003). The global DEMs, generated by Satellite Pour l'observation de la Terre -5 (SPOT-5), the advanced space-borne thermal emission and reflection radiometer (ASTER), and the shuttle radar topography mission (SRTM) are used in wide range of applications with different grid interval and accuracy (Yastikli et al., 2014).

The airborne light detection and ranging (LiDAR) and photogrammetry are the techniques for larger scale DSM generation offering higher ground sampling distance (GSD) and vertical accuracy. The LiDAR is collecting point clouds which include multi-returns from the visible surface of Earth together with the intensity values (Baltsavias, 1999). The data collection for DSM generation with LiDAR has been performed by multi sensor system includes LiDAR system, digital camera and GPS/IMU positioned on the same platform. The DSM with the point density of $10-16 \mathrm{pts} / \mathrm{m}^{2}$ can be produced with the LiDAR. The high production cost of LiDAR DSM is the main limitation in comparison to the photogrammetry.

The automatic DSM generation with the aerial images is also possible with stereo image matching which is originally introduced more than two decades ago (Heipke, 1996). In traditional approach, feature points are extracted first and then corresponding features are searched in the overlapping images with feature based matching for DSM generation (Haala, 2013). The similarity between pixels by comparing windows around pixels of interest is computed in correlation based stereo matching. The slanted surfaces and abrupt changes as caused by 
depth discontinuities, radiometric differences often occur due to different imaging characteristics of the camera, different exposure times, etc. are affecting the matching quality in traditional stereo image matching (Hirschmuüller, 2011). The traditional stereo image matching is also facing with the problem in urban and forest areas for automatic DSM generation because of repetitive pattern (Yastikli and Jacobsen, 2003). In contrast to the traditional approach, recent stereo matching algorithms is based on pixel-wise matching. The semiglobal matching (SGM) is a popular and well performing example of new generation image matching for dense DSM generation (Hirschmüller, 2008). The very high resolution DSMs which corresponds to the ground sample distance (GSD) of the original images can be generated with new pixel-wise matching approaches (Haala, 2013). The numbers of the research institutes and photogrammetric software vendors are currently developed software tools for dense DSM generation using the aerial images. The European Spatial Data Research Organisation (EuroSDR) started a follow up initiative to evaluate ongoing development in image based high resolution DSM generation in February, 2013 (Fritsch et al., 2013).

In this study, the performance validation of high resolution DSM generated by pixel-wise dense image matching in part of Istanbul is performed. The study area in Istanbul is including different land classes such as open areas, forest and built-up areas to test performance of dense image matching in different land classes. The overview of the SGM is presented in the sections to follow. The experimental studies that are presented in Section 3 provide the details about the performance validation of high resolution DSM generated by pixel-wise dense image matching over the test area in Istanbul. Section 4 concludes the paper.

\section{SEMI-GLOBAL MATCHING (SGM)}

SGM is the pixel-wise matching performed on image pairs using a Mutual Information (MI) based cost term for compensating radiometric differences (Hirschmüller and Tilman, 2010). The global cost function used in this approach is based on following equation (Hirschmüller, 2011):

$$
E(D)=\sum_{p}\left(C\left(p, D_{p}\right)+\sum_{q \in N_{p}} P_{1} T\left[\left|D_{p}-D_{q}\right|=1\right]+\sum_{q \in N_{p}} P_{2} T\left[\left|D_{p}-D_{q}\right|>1\right]\right)
$$

The cost function in equation 1 sums the matching cost $C$ over all pixel $p$, according the given disparities $D_{p}$ and adds a small penalty $P_{1}$ for neighboring pixels that have slightly different disparities (i.e. depths) and a large penalty $P_{2}$ for neighboring pixels with higher disparity differences. The goal is to find the disparity image $D$ that minimizes this function (Hirschmüller et al., 2012). In SGM one dimensional optimizations from all directions through the image have been performed as shown in Figure 1.

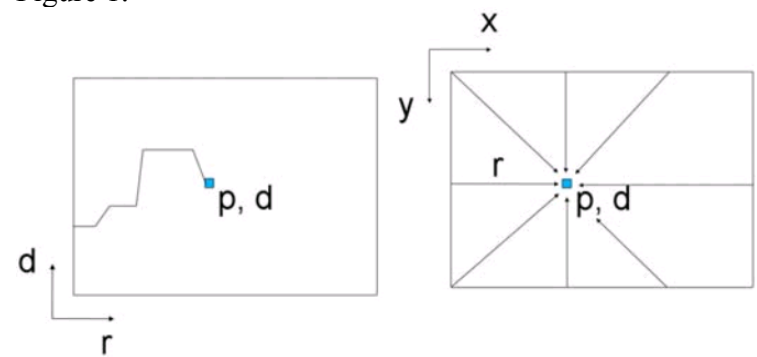

Figure 1. Eight optimization paths (Hirschmüller, 2011)
The disparity $d$ is chosen for each pixel $p$ where the sum of costs of paths that reach the pixel at the disparity from eight different directions $r$ is lowest. SGM results are fused by selecting the median disparity value for each pixel. The all disparity images pixels are reconstructed and re-projected into an equidistant grid for DSM generation (Hirschmüller and Tilman, 2010).

The main limitation of the SGM is its memory consumption that depends, as for all global methods, on the number of pixels and the disparity range (Hirschmüller et al., 2012). In aerial image processing, SGM requires a lot of processing time, but each image can be matched independently against its neighboring, overlapping images. Therefore, SGM based image processing can be implemented on a computer cluster (Hirschmüller, 2011). The SGM approach fulfils our need of high DSM resolution with using the multi-core CPUs as well as the graphics processing units (GPU) to accelerate DSM production.

\section{PERFORMANCE EVALUATION OF DSM GENERATED BY DENSE IMAGE MATCHING}

Software tools for dense image matching are currently developed by a number of research institutes and photogrammetric software vendors. The Microsoft UltraMap V3.0. Dense Matcher and Astrium GEO-Information Pixel Factory are commercially available software tolls high resolution DSMs generation based on pixel-wise dense image matching. The French National Mapping Agency (IGN) developed the software system MicMac realizing the multiimage matching by energy minimization either using graph cut or a modified dynamic programming/SGM approach. The German Aerospace Center (DLR) developed own software based on SGM algorithm. In this research, the performance of high resolution DSMs which were generated using the German Aerospace Center (DLR)'s own developed software as a research institution and Microsoft UltraMap V3.1 commercial software tool using the aerial images at GSD of $10 \mathrm{~cm}$.

\subsection{The Study Area and Data Set}

The study area is including the Zekeriyaköy district of Istanbul in Turkey located on European side of the city. The study area in Istanbul is including different land classes such as open areas, forest and built-up areas to test performance of dense image matching in different land classes (Figure 2).

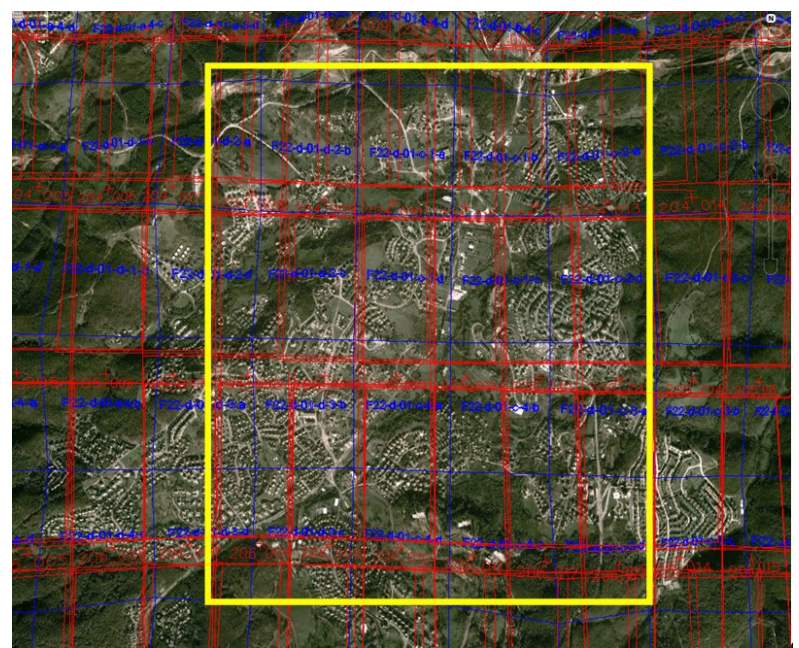

Figure 2. Study area in Istanbul. 
The aerial images, used in this study, were collected on September, 2013 over test area in Istanbul with the UltraCam $\mathrm{Xp}$ aerial full frame camera in the context of the $1 / 1000$ scaled photogrammetric map production project of Istanbul Metropolitan Municipality. The test sub-block was consist of the three overlapping strips with 18 images approximately $70 \%$ forward overlap and $35 \%$ side-lap at GSD of $10 \mathrm{~cm}$. The exterior orientation parameters of the images were acquired from bundle block adjustment of image block in the photogrammetric map production project. The forward overlap of $80 \%$ and side-lap of $60 \%$ is requested for pixel-wise dense image matching for high redundancy to eliminate wrong correspondences matching (Hirschmüller and Tilman, 2010). These images were available as tiled tiff uncompressed 8 bit/pix with 11310x 17310pix at 196Mpix/image. Since the Microsoft UltraMap software requires raw image data, the raw image data was also obtained from Istanbul Metropolitan Municipality. The LiDAR data covering the Istanbul test area was used as a reference surface which was acquired from Istanbul Metropolitan Municipality in 2013with point density of 16 $\mathrm{pts} / \mathrm{m} 2$.

\subsection{Performance Evaluation}

The high resolution DSMs generation has been performed with the German Aerospace Center (DLR)'s own developed software as a research institution and Microsoft UltraMap V3.1. commercial photogrammetric software (see Figure 3). The density of the generated high resolution DSMs of the test area was $100 \mathrm{pts} / \mathrm{m}^{2}$, which is equal to the original GSD $(10 \mathrm{~cm})$ of aerial images.

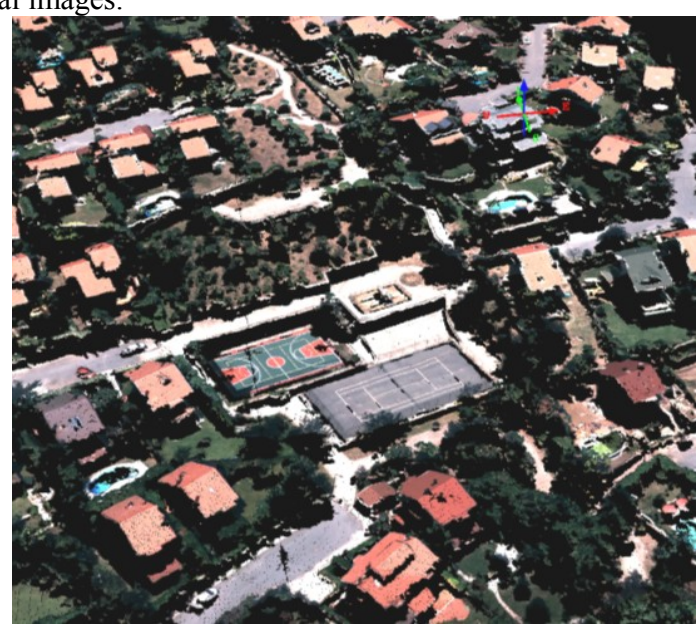

(a)

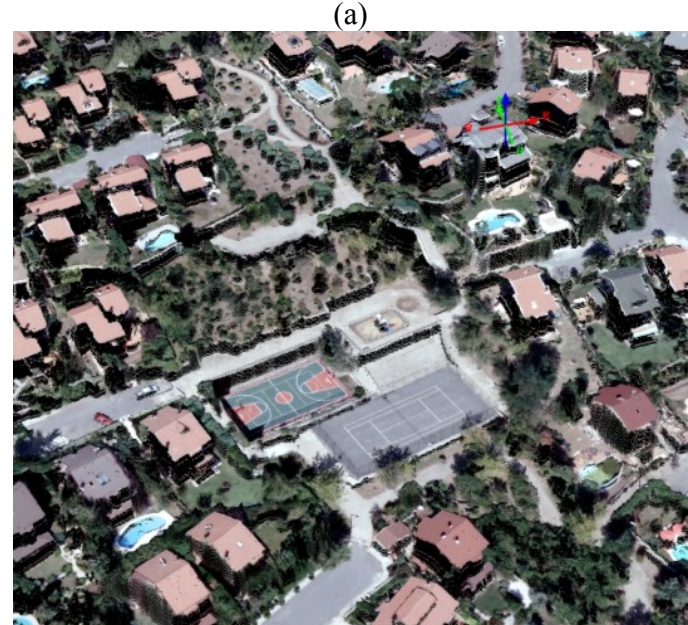

(b)

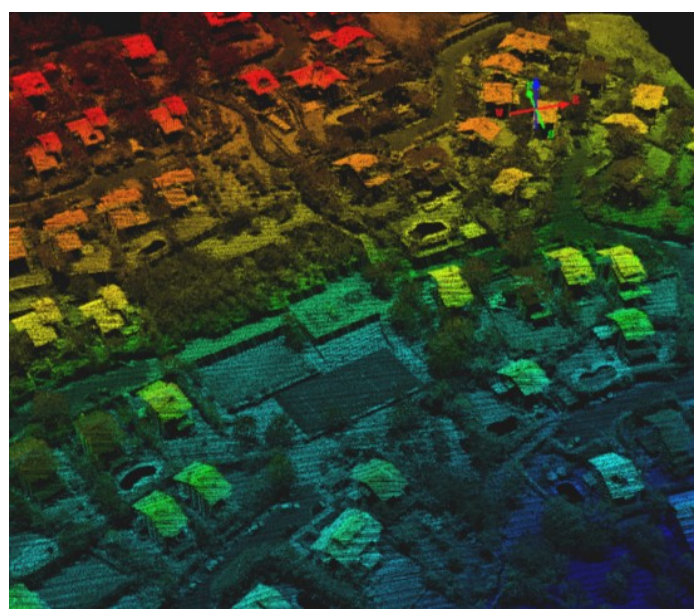

(c)

Figure 3. The generated DSM with UltraMap V3.1 (a), DLR software (b) and reference LiDAR point cloud (c)

The high resolution DSMs generation and performance evaluation have been performed as a part of the nation-wide dense DSM generation with point density of $100 \mathrm{pts} / \mathrm{m}^{2}$ and true orthophoto generation project in Turkey. The Yildiz Technical University serves as consultant of Ministry of Environment and Urbanism of Turkey in this project. The performance evaluation of generated high resolution DSMs have been performed by comparing with reference LiDAR DSM. To test the new pixelwise dense image matching on different land classes, performance evaluation was performed separately on open areas, forest, and built-up areas. The differences are depicted in Figure 4 for DLR DSM (a) and UltraMap DSM (b) in urban area.

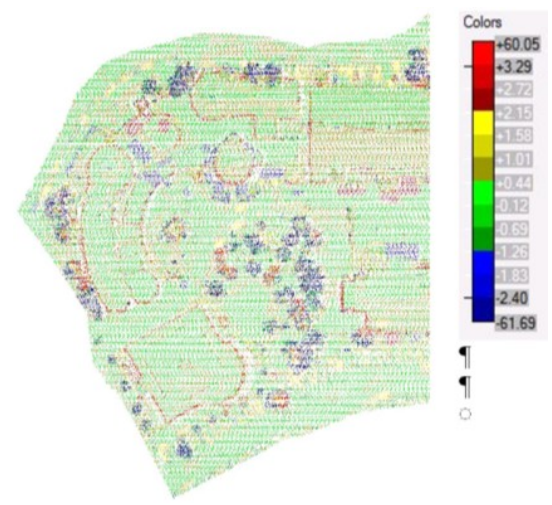

(a)

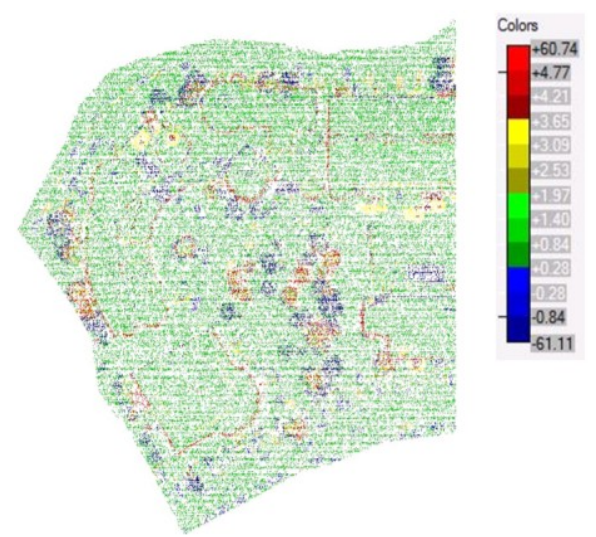

(b)

Figure 4. The computed elevation differences for DLR DSM (a) and UltraMap DSM (b) in urban 
Table 1 shows the standard deviations and root mean square errors (RMSE) of differences for different land classes. The differences larger than three sigma were eliminated and the root mean square errors $\left(\mathrm{RMSE}_{\mathrm{Z}}\right)$ and standard deviations of differences were recomputed (see Table 2).

Table 1. Standard deviations and (RMSE) of differences for selected areas

\begin{tabular}{|l|c|c|c|c|}
\hline \multirow{2}{*}{$\begin{array}{l}\text { Land } \\
\text { classes }\end{array}$} & \multicolumn{2}{|c|}{ DLR DSM } & \multicolumn{2}{c|}{ UltraMap DSM } \\
\cline { 2 - 5 } & $\begin{array}{c}\text { Standard } \\
\text { deviation }\end{array}$ & RMSE $_{Z}$ & $\begin{array}{l}\text { Standard } \\
\text { deviation }\end{array}$ & RMSE $_{Z}$ \\
\hline Open area & 0.96 & 0.983 & 0.906 & 1.332 \\
Urban Area & 2.074 & 2.088 & 2.008 & 2.325 \\
Forest & 5.382 & 6.177 & 4.552 & 5.563 \\
\hline
\end{tabular}

Table 2 Standard deviations and (RMSE) of differences for selected areas after elimination differences higher than 3 sigma

\begin{tabular}{|l|c|c|c|c|}
\hline \multirow{2}{*}{$\begin{array}{l}\text { Land } \\
\text { classes }\end{array}$} & \multicolumn{2}{|c|}{ DLR DSM } & \multicolumn{2}{c|}{ UltraMap DSM } \\
\cline { 2 - 5 } & $\begin{array}{c}\text { Standard } \\
\text { deviation }\end{array}$ & RMSE $_{Z}$ & $\begin{array}{c}\text { Standard } \\
\text { deviation }\end{array}$ & RMSE $_{Z}$ \\
\hline Open area & 0.316 & 0.431 & 0.347 & 1.048 \\
Urban Area & 1.597 & 1.610 & 1.487 & 1.802 \\
Forest & 4.613 & 5.322 & 3.812 & 4.807 \\
\hline
\end{tabular}

As can be seen in Table 2, standard deviations of differences for open areas and urban areas is close to each other as a result of comparison between the generated DLR DSM and UltraMap DSM in comparison to the reference LiDAR DSM. The main difference was in forest area in Table 2. In order to further quantitative analyses of the DSM differences, elevation profiles were extracted for selected areas. These lines of interest are overlaid to the orthoimages in the Figure 5. The profile line numbered with label 1 is extracted from the respective surface models at the flat road surface. Profile line 2 is cover inclined terrain including tree. Profile 3 has its starting point close to a building and then crosses building. The resulting height profiles which have different terrain characteristics are shown in Figure 6.

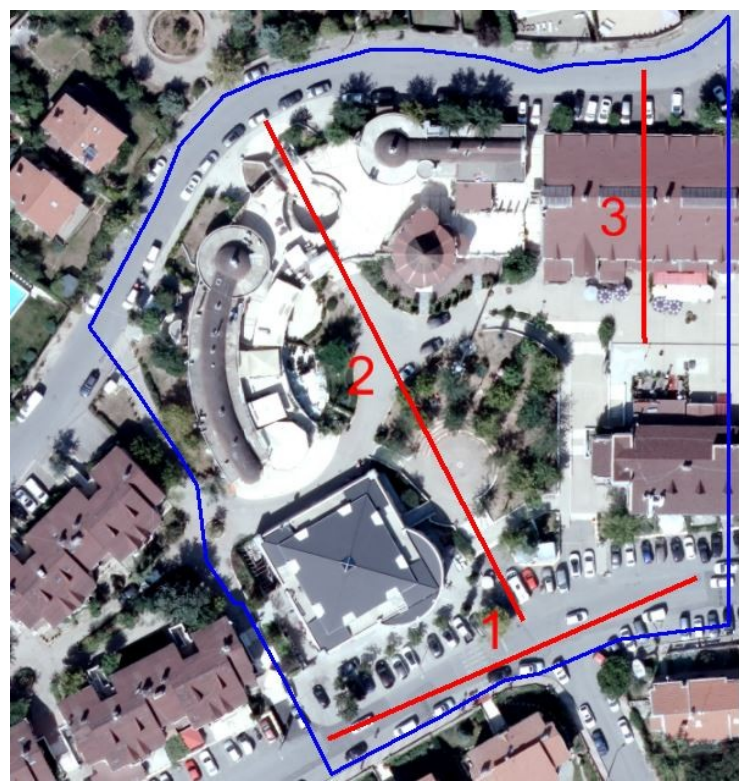

Figure 5. The elevation profiles

Profiles extracted for UltraMap DSM (blue line) apparently are higher than the reference LiDAR DSM (green line). Furthermore, the profile from the UltraMap DSM is relatively noisy for all profiles. Profiles extracted for DLR DSM (red line) are coherent with the reference LiDAR DSM. The noisy characteristics in LiDAR DSM can be seen in at Profile 2 at the area covered with tree because of the multiple returns of LiDAR pulse. Because of that, standard deviation in Table 2 is high for the forest.

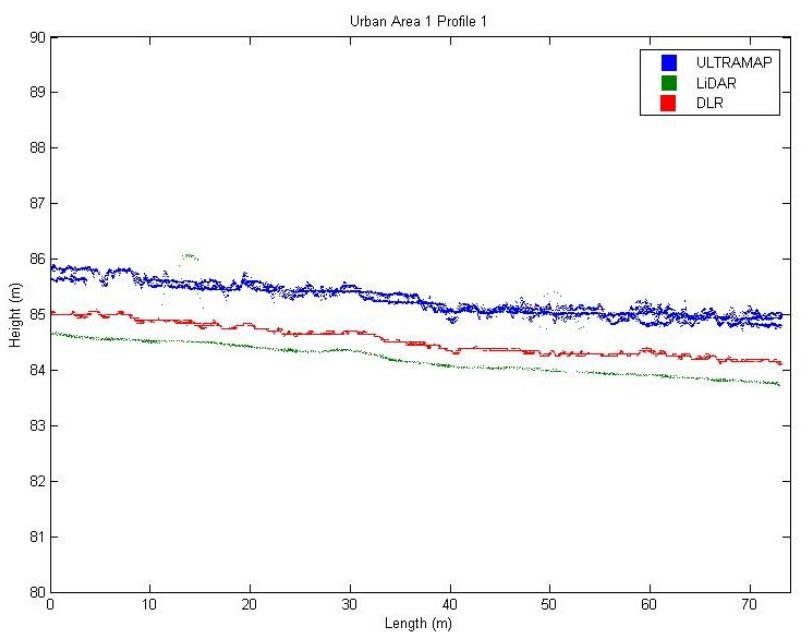

(a)

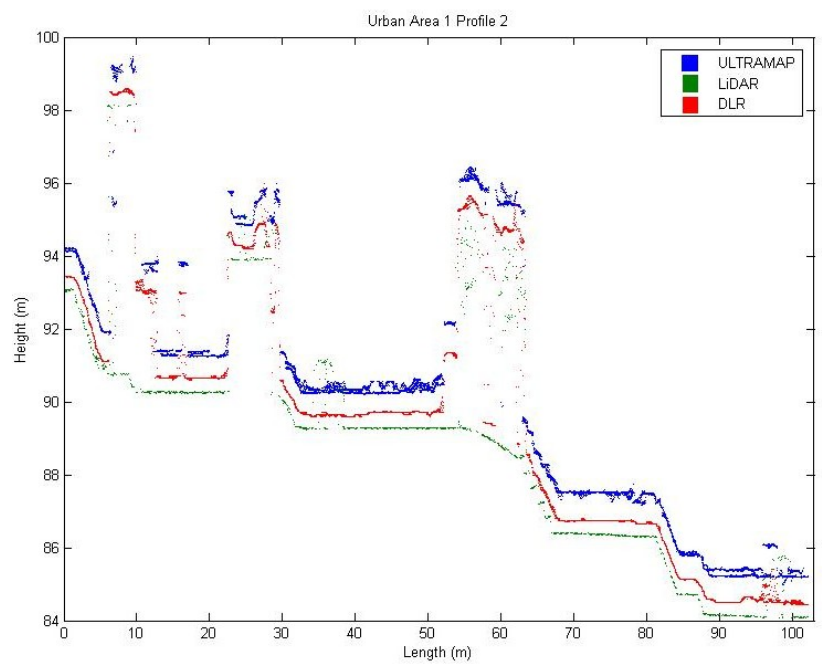

(b)

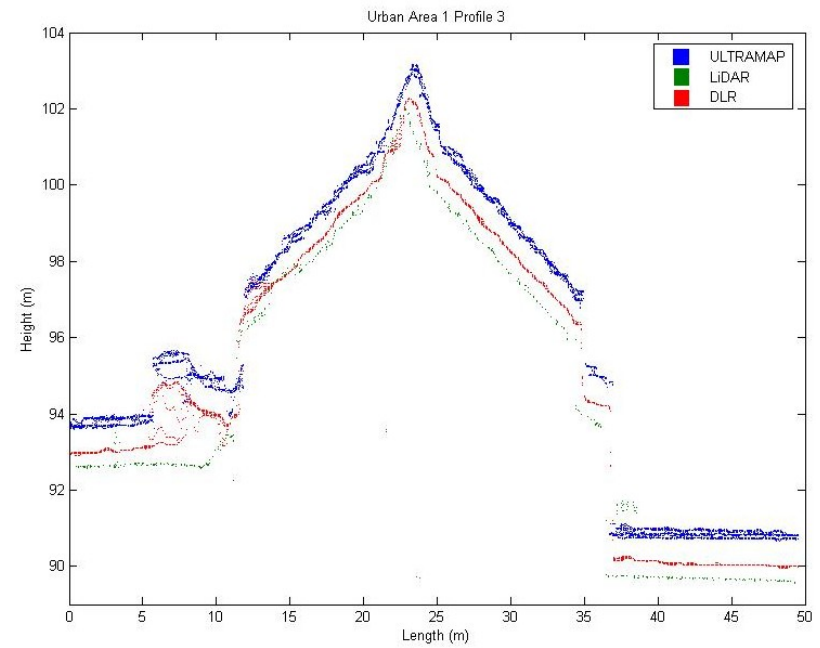

(c)

Figure 6. DSM profiles at flat road surface (a), inclined terrain including tree (b) and build up area (c) 


\section{CONCLUSIONS}

In this study, high resolution DSMs were generated by pixelwise dense image matching using the German Aerospace Center (DLR) research institute software's and Microsoft UltraMap V3.1 commercial software in the Istanbul test area including various land classes. The performance evaluation of the generated high resolution DSMs with the point density of 100 $\mathrm{pts} / \mathrm{m}^{2}$ resulted almost same accuracy for both DLR and UltraMap DSMs for open and urban areas against the reference LiDAR DSM. The small differences at $\mathrm{RMSE}_{\mathrm{Z}}$ and standard deviations of differences can be seen on forest area.

The obtained result from Istanbul test area showed that, high resolution DSM with density on $10 \mathrm{~cm}$ which corresponds to the ground sample distance (GSD) of original aerial image can be generated successfully by pixel-wise dense image matching using commercial and research institution's software. The DSMs generations have been achieved successfully urban structure and forest where the traditional image matching algorithms face with the problems. The new pixel-wise matching approaches can be used for the nation-wide high resolution DSM and true orthophoto generation for wide range of applications.

\section{ACKNOWLEDGEMENTS (OPTIONAL)}

The authors would like to thank the Metropolitan Municipality of Istanbul for supplying the reference LiDAR data set and aerial images covering the Istanbul test area.

\section{REFERENCES}

Baltsavias, E. P., 1999. Airborne Laser Scanning: Existing Systems And Firms And Other Resources. In: ISPRS Journal of Photogrammetry and Remote Sensing, 54, pp. 164-198.

Fritsch, D., Pfeifer, N. \& Franzen, M. (Eds.)., 2013. Proceedings of the 2nd EuroSDR workshop on High Density Image Matching for DSM Computation, EuroSDR Publication Series, No. 63.

Haala, N., 2013. The Landscape of Dense Image Matching Algorithms. In: Fritsch, D. (Ed.): Photogrammetric Week '13, Wichmann, Berlin/Offenbach, 271-284.

Heipke, C., 1996. Overview of image matching techniques. In: OEEPE Workshop on the Application of Digital Photogrammetric Workstation, OEEPE Official Publication No.33, pp.173-189.

Hirschmüller, H., 2008. Stereo Processing by Semi-Global Matching and Mutual Information. In: IEEE Transactions onPattern Analysis and Machine Intelligence, Volume 30(2), February 2008, pp. 328-341.

Hirschmüller, H., and Tilman B., 2010. Evaluation of Digital Surface Models by Semi-Global Matching, $D G P F$ 2010, 01-02 July 2010, Vienna, Austria.

Hirschmüller, H., Buder M., and Ernst I., 2012. Memory Efficient Semi-Global Matching. In: SPRS Annals of the Photogrammetry, Remote Sensing and Spatial Information Sciences, Volume I-3, August 2012 in Melbourne, Australia.
Hirschmuüller, H., 2011. Semi-Global Matching - motivation, developments and applications. In: Photogrammetric Week 11, Fritsch, D. (ed), Wichmann, Heidelberg, pp. 173-184.

Jacobsen, K, 2003. DEM Generation from Satellite Data. In: EARSeL Workshop, 5-7 June, Ghent, Belgium, pp. 273-276.

Kaczynski, R., Majde, A., Ewiak, I., 2004. Accuracy of DTM and Ortho generated from Ikonos Stereo Images. In: ISPRS Congress, Geo-Imagery Bridging Continents, Istanbul (on CDROM).

Li, R., Zhou, G., Schmidt, N. J. et al., 2002. Photogrammetric processing of high-resolution airborne and satellite linear array ste-reo images for mapping applications. In: International Journal of Remote Sensing, 23(20): 4451-4473. doi: 10.1080/ 01431160110107662

Sefercik, U. G., Yastikli, N., 2014. Assessment Of Interferometric Dems From Terrasar-X Stripmap And Spotlight Stereopairs: Case Study In Istanbul. In: The Photogrammetric Record, Volume: 29 Issue: 146 Pages: 224-240 Published: Jun 2014.

Sefercik, U. G., Yastikli, N., Dana I., 2014. DEM Extraction In Urban Areas Using High-Resolution TerraSAR-X Imagery. In: Journal of the Indian Society of Remote Sensing, Springer (ISI), Doi: 10.1007/s12524-013-0317-9, Volume: 42 Issue: 2 Pages: 279-290 Published: JUN 2014.

Toutin, T., 2002. DEM from stereo Landsat 7 ETM+ data over high relief areas. In: International Journal of Remote Sensing, 23(10): 2133-2139. doi: 10.1080/01431160110098005

Toutin, T., 2008. ASTER DEMs for geomatic and geoscientific applications: A review. In: International Journal of Remote Sensing, 29(7): 1855-1875. doi: 10.1080/01431160701408477

Yastikli, N., Jacobsen, K., 2003. Automatic Digital Elevation Model Generation, Problem and Restrictions in Urban Areas. In: Journal of Yildiz Technical University, ISSN: 1300-2120, Sayı: 2003/2.

Yastikli, N., Sefercik, U. G. and Esirtgen, F., 2014. Quantitative Assessment of Remotely Sensed Global Surface Models Using Various Land Classes Produced from Landsat Data in Istanbul. In: Chinese Geographical Science, Volume: 24, Issue: 3, Pages: 307-316, Published: Jun 2014. 